\title{
Magnetic Resonance Spectroscopy and Single-Photon Emission Computed Tomography in the Evaluation of Cerebral Tumors: A Case Report
}

\author{
Ioannis Siasios ${ }^{\mathrm{a}, \mathrm{b}, \mathrm{g}}$, Varvara Valotassiou ${ }^{\mathrm{c}}$, Eftychia Kapsalaki ${ }^{\mathrm{d}}$, Ioannis Tsougos ${ }^{\mathrm{e}}$, \\ Panagiotis Georgoulias $^{c}$, Aggeliki Fotiadou ${ }^{a}$, Maria Ioannou ${ }^{f}$, Georgios Koukoulis ${ }^{f}$, \\ Vassilios Dimopoulos ${ }^{\mathrm{b}}$, Kostas Fountas ${ }^{\mathrm{a}}$
}

\begin{abstract}
In their daily clinical practice, physicians have to confront diagnostic dilemmas which cannot be resolved by the application of only one imaging technique. In this case report, we present a 66 -year-old woman who was admitted to our institution for the surgical resection of a recently diagnosed brain tumor. The patient had a history of epileptic seizures and was hospitalized in the past for anti-phospholipid syndrome related to a non-Hodgkin lymphoma in remission. Magnetic resonance imaging (MRI) examination revealed an enhancing right parasagittal lesion with significant edema suggestive of a high grade glioma. Advanced MRI techniques including proton magnetic resonance spectroscopy $\left({ }^{1} \mathrm{H}-\mathrm{MRS}\right)$ showed findings compatible of glioma. An additional examination was performed as part of a protocol that we are routinely performing in our institution for all brain tumors including not only the gold standard advanced MRI techniques but also single-photon emission computed tomography (SPECT) with technetium-99m (Tc99m). Brain SPECT indicated the presence of a meningioma which was verified by the histopathology of the resected specimen. In conclusion, a multimodality approach for the pre-surgical assessment of brain tumors has significant advantages not only for the diagnosis but also for the evaluation of intracranial tumors histology.
\end{abstract}

Keywords: Brain tumors; MRI advanced techniques; ${ }^{1} \mathrm{H}-\mathrm{MRS}$; Brain SPECT; Tumor grading; Pre-surgical evaluation

Manuscript accepted for publication October 21, 2016

a Department of Neurosurgery, University Hospital of Larissa, Greece ${ }^{b}$ Department of Neurosurgery, Jacobs School of Medicine and Biomedical Sciences, University at Buffalo, State University of New York, Buffalo, Buffalo, NY, USA

'Department of Nuclear Medicine, University Hospital of Larissa, Greece ${ }^{\mathrm{d} D e p a r t m e n t}$ of Diagnostic Radiology, University Hospital of Larissa, Greece 'Department of Medical Physics, University Hospital of Larissa, Greece fDepartment of Pathology, University Hospital of Larissa, Greece

'Corresponding Author: Ioannis Siasios, University at Buffalo Neurosurgery, 100 High Street, Suite B4, Buffalo, NY 14203, USA.

Email: isiasios@ubns.com

doi: https://doi.org/10.14740/jocmr2775w

\section{Introduction}

Magnetic resonance spectroscopy (MRS), firstly introduced for clinical use in 1990, was recognized as one of the most promising tools in detecting metabolic activity profiles that could help in non-invasive histological discrimination of brain tumors [1]. Since then and for the last decades, MRS combined with other magnetic resonance imaging (MRI) advanced techniques such as perfusion-weighted imaging (PWI) and diffusion-weighted imaging (DWI) has become a routine presurgical evaluation method of intracranial tumors [2-10]. In addition, MRS is popularized among scientists as a valuable diagnostic modality in several other pathological conditions such as hypoxia-ischemia in neonates, head trauma, epilepsy, leukodystrophies and metabolic diseases [11, 12].

The definition of tumor histology and grade is very important for the treatment strategizing. Despite its high performance, MRS solely or in combination with other advanced MRI techniques cannot resolve differential diagnostic dilemmas in some cases. A possible solution to this problem would be the supplemental use of other imaging modalities which could contribute significantly to the recognition of the tumor histopathological profile.

In this case report, we present an interesting case of a 66-year-old woman who had a supratentorial intracranial lesion with MRI characteristics similar to glioma. The combination of MRS and single-photon emission computed tomography (SPECT) with technetium-99m (Tc99m) led to the pre-operative diagnosis of a meningioma which was confirmed by the histopathological analysis of the tumor specimen.

\section{Case Report}

A 66-year-old woman was admitted to our hospital for scheduled resection of a right parasagittal brain lesion. She had a history of epileptic seizures and anti-phospholipid syndrome related to a non-Hodgkin lymphoma in remission. The patient underwent brain MRI, ${ }^{1} \mathrm{H}-\mathrm{MRS}$ and brain SPECT as part of a protocol which was based on the collaboration of the Departments of Medical Physics, Radiology and Neurosurgery aiming to study the combination of brain SPECT and 
Table 1. The Ratios of NAA/Cr, Chol/Cr, and Chol/NAA According to the Performed ${ }^{1} \mathrm{H}-\mathrm{MRS}$ Study

\begin{tabular}{llll}
\hline & Lesion site (right hemisphere) & Normal brain tissue (left hemisphere) & Deviation \\
\hline NAA/Cr & 1.56 & 1.63 & $(-) 4$ \\
Chol/Cr & 4.05 & 1.15 & $(+) 252$ \\
Chol/NAA & 2.03 & 0.709 & $(+) 186$ \\
\hline
\end{tabular}

Chol: choline; Cr: creatinine; NAA: N-acetylaspartate.

MRI advanced techniques in the amelioration of brain tumor diagnosis. A 3-Tesla MRI scanner (GE, Healthcare, Signa ${ }^{\circledR}$ HDx) was used. ${ }^{1} \mathrm{H}-\mathrm{MRS}$ study was feasible through two different spectroscopy imaging protocols: proton brain exam (PROBE) single voxel (SV) and two-dimensional multivoxel (2D chemical shift imaging (CSI)) spectroscopy. The used sequence was point-resolved spectroscopy (PRESS) pulse with phase encoding gradients towards two directions, automatic and manual shimming and Gaussian water suppression. A single voxel with a volume less than $3.4 \mathrm{~mL}$ was cautiously obtained from the tumor area away from the tumor margins by applying measurements of 1,500/35 m/s (TR/TE) and using 128 signal acquisitions (Nacq). 2D CSI technique was applied to the measurements of 1,000/144 m/s (TR/TE) by using $16 \times 16$ phase encoding steps, with a thickness of $10 \mathrm{~mm}$ based on the patient's intracranial topography. The duration of spectroscopy in both imaging protocols did not exceed the 4- 5 min. Besides the tumor area, spectroscopic images were obtained also from the healthy brain tissue (Table 1). The localization of the desirable voxel area was retrospectively feasible through sagittal, axial and coronal images with T1weighted FSE, T2-weighted FLAIR and T2-weighted imaging sequences. Contrast agent was used after the completion of the spectroscopy for the optimization of our surgical plan- ning as these images were intergrated to our neuronavigation system.

The brain SPECT was performed 20 - 30 min after intravenous injection of $925 \mathrm{MBq}(25 \mathrm{mCi})$ tracer activity. The Myoview $^{\mathrm{TM}}$ (General Electric Healthcare Ltd) was used for the preparation of the radiopharmaceutical which reconstituted with Tc- $99 \mathrm{~m}$ pertechnetate $\left(99 \mathrm{mTcO}_{4}\right)$ sterile solution in the Department of Nuclear Medicine. The processing and fusion of the obtained images was performed through the Xeleris Workstation (GE Healthcare).

According to conventional MRI examination, an enhancing right parasagittal brain lesion with significant edema was recognized. MRS examination raised the suspicion of glioma based on the metabolic profile at the center of the tumor which indicated high ratio of choline to $\mathrm{N}$-acetylaspartate (Chol/ NAA) (Figs. 1, 2). The SPECT examination indicated intense and homogenous uptake of the radiopharmaceutical, with notably high standardized uptake value (SUV), supporting the diagnosis of meningioma (Fig. 3). The patient underwent a right fronto-parietal craniotomy and her tumor was completely resected. The histopathological analysis of our specimen indicated the diagnosis of a meningioma grade I (Figs. 4, 5). The patient had an uneventful postoperative course and at her last follow-up she is tumor free.

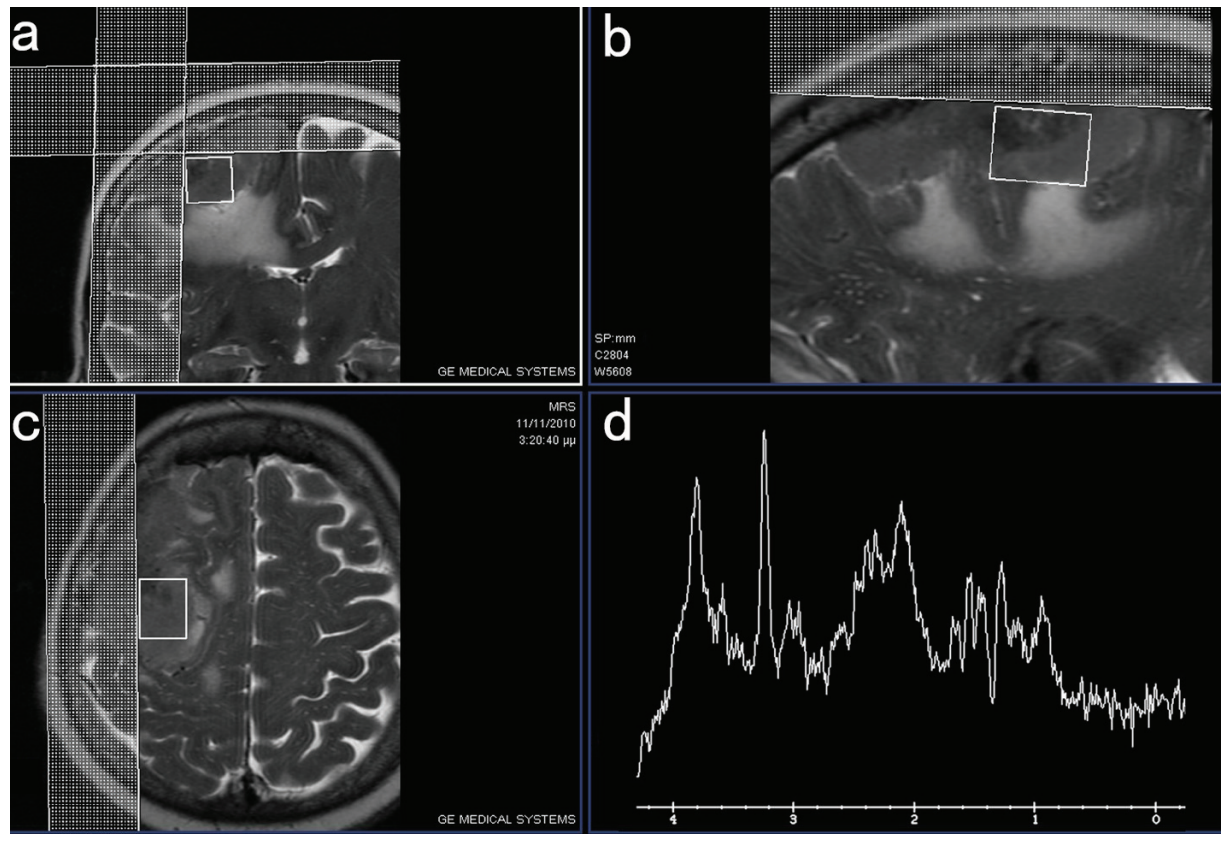

Figure 1. MRI images in coronal (a), sagittal (b) and axial plane (c) demonstrating the area of the tumor where the voxel of the performed MRS study (d) was obtained. 


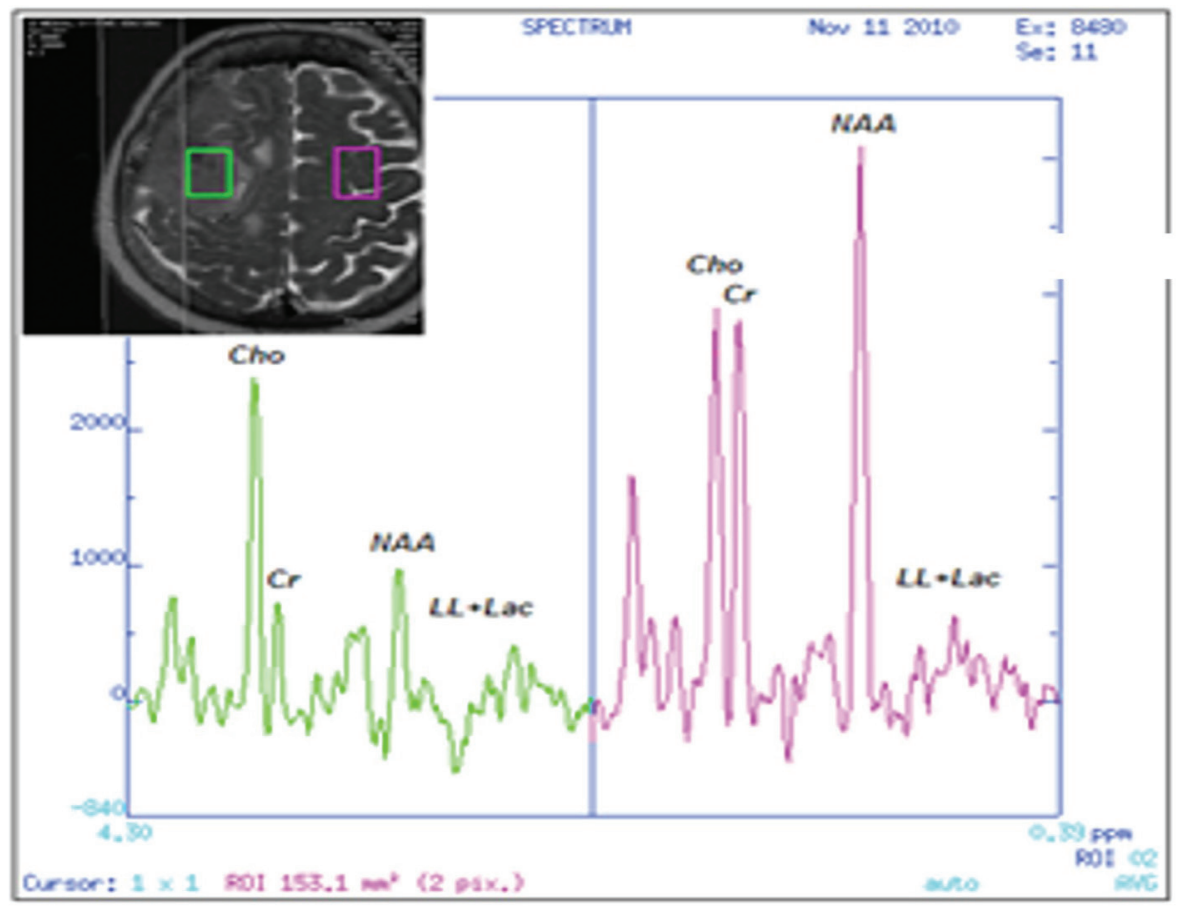

Figure 2. ${ }^{1} \mathrm{H}-\mathrm{MRS}$ of the tumor area (left) showing the high Chol/NAA ratio in comparison to the normal MRS findings of the opposed normal brain tissue (right).

\section{Discussion}

In vivo MRS is a well-known imaging MRI technique for the evaluation of tumor histology and grade. The accurate prediction of tumor type based on ${ }^{1} \mathrm{H}-\mathrm{MRS}$ can reach $82.5 \%$ and according to the literature, this technique has $100 \%$ sensitivity and more that $90 \%$ specificity as a diagnostic method [13]. It is based on the detection of specific metabolites such as Chol, NAA, glutamate (Glu), glutamine (Gln), creatinine (Cr), myoinositol, lactate and lipids [14-18]. NAA is synthesized in the neuronal mitochondria and transported into the cytosol, as well as along the axons of the neurons. It is present primar-

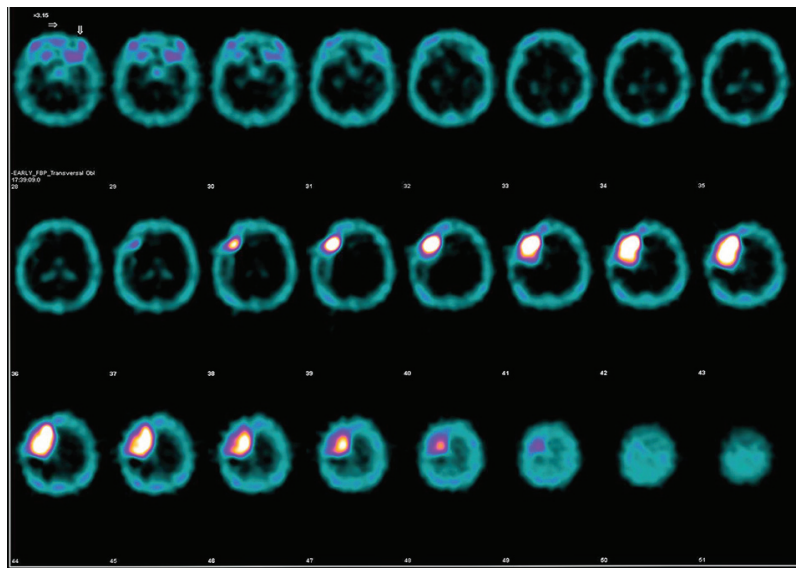

Figure 3. Axial images of the performed brain SPECT with technetium$99 \mathrm{~m}$ (Tc99m) demonstrating high standardized uptake value (SUV). ily in neuronal tissue, thus it is used as a marker of neuronal density and integrity. NAA reduction is observed in neuronal cell death usually seen in gliomas with grade II or more, glioblastomas, lymphomas, metastasis and gliomatosis, whereas in extra-axial tumors, such as meningiomas and schwannomas, NAA is usually absent $[14,16-18]$. Chol is involved in cell membrane phospholipids' synthesis-degradation and its levels are raised in increased cell membrane metabolism or abnormal neuronal cell increase [14, 16-18]. Cr/phosphocreatine is involved in cell energy capacity and decreases in any pathological condition associated with increased metabolic activity

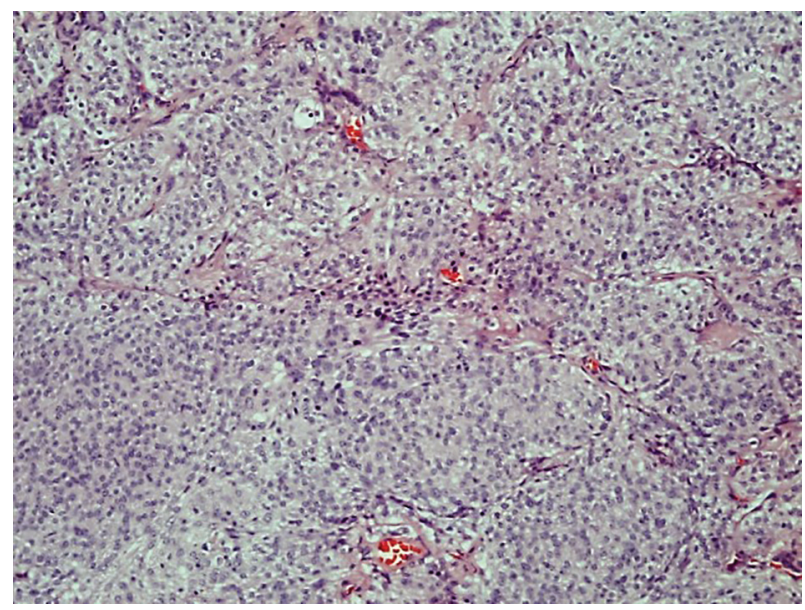

Figure 4. The tumor cells form lobules some partly demarcated by thin collagenous septae (hematoxylin and eosin stain, original magnification $\times 10)$. 


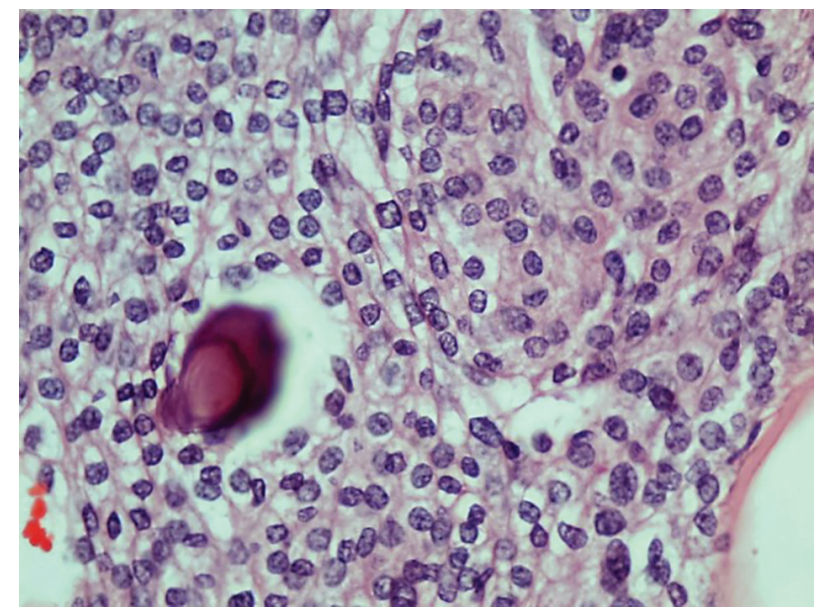

Figure 5. The tumor cells are uniform with oval nuclei with delicate chromatin that on occasion show central clearing or the formation of nuclear inclusions. Psammoma bodies are present (hematoxylin and eosin stain, original magnification $\times 40$ ).

$[14,16-18]$. Glx is the composite of Glu, Gln and gamma-aminobutyric acid (GABA) peaks. Glu is the most prevalent excitatory neurotransmitter in the brain and the precursor of the inhibitory neurotransmitter GABA [14, 16-18]. Myo-inositol is a specific glial marker as it can be found only in glial cells and subsequently in glial tumors where it is increased [14, 16-18]. Lactate is a marker of anaerobic glycolysis and its presence is detected in hypoxic and ischemic conditions, as well as in lesions with high rate of glycolysis and its elevation denotes higher tumor grade [14, 16-18]. At last, free lipids are observed in brain tissue necrosis and their presence is compatible with high grade malignant tumors, metastatic lesions, lymphomas and less with meningiomas [14, 16-18]. The metabolic profile of the brain lesion in our case according to the performed MRS study was characterized by high Chol/NAA ratio, a small increased concentration of lipids and lactate acid, high levels of myo-inositol and finally a small concentration of amino-acids. These findings were suggestive of a glioma.

Brain SPECT with Tc-99m was introduced in 1960s showing markedly the location of the tumor by demonstrating the disruption of the blood barrier in the pathological area [19]. Several radiopharmaceutical agents have been used until today such as Tc-99m diethylenetriaminepentaacetic acid (DTPA), Tc-99m glucoheptonate (Tc-99m GHA), thallium-201, Tc99m tetrofosmin, and Tc-99m sestamibi [19]. The last decades this modality was repeatedly implemented for the detection of tumor recurrence or remnants in high grade gliomas and for brain tumors grading [19-23]. Furthermore, it was proved of significant value in the differentiation between tumor necrosis due to irradiation and recurrence in surgically treated high grade gliomas [24]. In our case, brain SPECT showed intense and homogenous uptake of the radiopharmaceutical with high SUV indicating the presence of a meningioma. According to the reported cases series in the literature, brain SPECT can help in the differentiation between gliomas and benign tumors and between typical and atypical meningiomas [22, 25].

In conclusion, ${ }^{1} \mathrm{H}-\mathrm{MRS}$ with the supplemental informa- tion of the brain SPECT provided an accurate pre-surgical diagnosis in our case resolving a significant diagnostic dilemma. It seems that a multi-modality approach in the diagnosis of brain tumors would be of great benefit for the patients and also for the physicians who are involved in patient's treatment planning.

\section{Conflicts of Interest}

None.

\section{Financial Support}

None.

\section{References}

1. Bottomley PA. Human in vivo NMR spectroscopy in diagnostic medicine: clinical tool or research probe? Radiology. 1989;170(1 Pt 1):1-15.

2. Guzman-De-Villoria JA, Mateos-Perez JM, FernandezGarcia P, Castro E, Desco M. Added value of advanced over conventional magnetic resonance imaging in grading gliomas and other primary brain tumors. Cancer Imaging. 2014;14:35.

3. Hollingworth W, Medina LS, Lenkinski RE, Shibata DK, Bernal B, Zurakowski D, Comstock B, et al. A systematic literature review of magnetic resonance spectroscopy for the characterization of brain tumors. AJNR Am J Neuroradiol. 2006;27(7):1404-1411.

4. Julia-Sape M, Griffiths JR, Tate AR, Howe FA, Acosta D, Postma G, Underwood J, et al. Classification of brain tumours from MR spectra: the INTERPRET collaboration and its outcomes. NMR Biomed. 2015;28(12):17721787.

5. Moussazadeh N, Tsiouris AJ, Ramakrishna R. Advanced Imaging for Biopsy Guidance in Primary Brain Tumors. Cureus. 2016;8(2):e504.

6. Orphanidou-Vlachou E, Auer D, Brundler MA, Davies NP, Jaspan T, MacPherson L, Natarajan K, et al. (1)H magnetic resonance spectroscopy in the diagnosis of paediatric low grade brain tumours. Eur J Radiol. 2013;82(6):e295301.

7. Rao PJ, Jyoti R, Mews PJ, Desmond P, Khurana VG. Preoperative magnetic resonance spectroscopy improves diagnostic accuracy in a series of neurosurgical dilemmas. Br J Neurosurg. 2013;27(5):646-653.

8. Rehman L, Rehman UL, Azmat SK, Hashim AS. Magnetic Resonance Spectroscopy: Novel Non-invasive Technique for Diagnosing Brain Tumors. J Coll Physicians Surg Pak. 2015;25(12):863-866.

9. Seeger A, Braun C, Skardelly M, Paulsen F, Schittenhelm J, Ernemann U, Bisdas S. Comparison of three different MR perfusion techniques and MR spectroscopy for multiparametric assessment in distinguishing recurrent 
high-grade gliomas from stable disease. Acad Radiol. 2013;20(12):1557-1565.

10. Shiroishi MS, Panigrahy A, Moore KR, Nelson MD, Jr., Gilles FH, Gonzalez-Gomez I, Bluml S. Combined MRI and MRS improves pre-therapeutic diagnoses of pediatric brain tumors over MRI alone. Neuroradiology. 2015;57(9):951-956.

11. Oz G, Alger JR, Barker PB, Bartha R, Bizzi A, Boesch C, Bolan PJ, et al. Clinical proton MR spectroscopy in central nervous system disorders. Radiology. 2014;270(3):658679.

12. Ratai EM, Gilberto Gonzalez R. Clinical magnetic resonance spectroscopy of the central nervous system. Handb Clin Neurol. 2016;135:93-116.

13. Server A, Josefsen R, Kulle B, Maehlen J, Schellhorn T, Gadmar O, Kumar T, et al. Proton magnetic resonance spectroscopy in the distinction of high-grade cerebral gliomas from single metastatic brain tumors. Acta Radiol. 2010;51(3):316-325.

14. Soares DP, Law M. Magnetic resonance spectroscopy of the brain: review of metabolites and clinical applications. Clin Radiol. 2009;64(1):12-21.

15. Sibtain NA, Howe FA, Saunders DE. The clinical value of proton magnetic resonance spectroscopy in adult brain tumours. Clin Radiol. 2007;62(2):109-119.

16. Callot V, Galanaud D, Le Fur Y, Confort-Gouny S, Ranjeva JP, Cozzone PJ. (1)H MR spectroscopy of human brain tumours: a practical approach. Eur J Radiol. 2008;67(2):268-274.

17. Chaumeil MM, Lupo JM, Ronen SM. Magnetic Resonance (MR) Metabolic Imaging in Glioma. Brain Pathol. 2015;25(6):769-780.

18. Hall H, Cuellar-Baena S, Dahlberg C, In't Zandt R, Den- isov V, Kirik D. Magnetic resonance spectroscopic methods for the assessment of metabolic functions in the diseased brain. Curr Top Behav Neurosci. 2012;11:169-198.

19. Alam SS, Junaid S, Ahmed SM. Evaluation of Technetium-99m glucoheptonate single photon emission computed tomography for brain tumor grading. Asian J Neurosurg. 2016;11(2):118-128.

20. Singh B, Kumar N, Sharma S, Watts A, Hazari PP, Rani $\mathrm{N}$, Vyas S, et al. 99mTc-MDM Brain SPECT for the Detection of Recurrent/Remnant Glioma-Comparison With ceMRI and 18F-FLT PET Imaging: Initial Results. Clin Nucl Med. 2015;40(10):e475-479.

21. Alexiou GA, Fotopoulos AD, Papadopoulos A, Kyritsis AP, Polyzoidis KS, Tsiouris S. Evaluation of brain tumor recurrence by $(99 \mathrm{~m}) \mathrm{Tc}-$ tetrofosmin SPECT: a prospective pilot study. Ann Nucl Med. 2007;21(5):293-298.

22. Fotopoulos AD, Kyritsis AP, Tsiouris S, Al-Boucharali J, Papadopoulos A, Voulgaris S, Alexiou GA. Characterization of intracranial space-occupying lesions by $(9)(9)(\mathrm{m})$ Tc-Tetrofosmin SPECT. J Neurooncol. 2011;101(1):8389.

23. Santra A, Kumar R, Sharma P. Use of 99m-technetiumglucoheptonate as a tracer for brain tumor imaging: An overview of its strengths and pitfalls. Indian J Nucl Med. 2015;30(1):1-8.

24. Zhang H, Ma L, Wu C, Xu BN. Performance of SPECT in the differential diagnosis of glioma recurrence from radiation necrosis. J Clin Neurosci. 2015;22(2):229-237.

25. Fotopoulos AD, Alexiou GA, Goussia A, Papadopoulos A, Kyritsis AP, Polyzoidis KS, Voulgaris S, et al. (99m) Tc-Tetrofosmin brain SPECT in the assessment of meningiomas-correlation with histological grade and proliferation index. J Neurooncol. 2008;89(2):225-230. 